\title{
Silencing of Rac3 Inhibits Proliferation and Induces Apoptosis of Human Lung Cancer Cells
}

\author{
Tie-Qin Liu ${ }^{1}$, Ge-Bang Wang ${ }^{1}$,Zheng-Jun Li², Xiang-Dong Tong ${ }^{3}$,Hong-Xu Liu ${ }^{1 *}$
}

\begin{abstract}
Background: Rac3, a member of the Rac family of small guanosine triphosphatases (GTPases), regulates a variety of cell functions, including the organization of the cytoskeleton, cell migration, and invasion. Overexpression of Rac3 has been reported in several human cancers. However, the role of Rac3 in lung cancer (LC) has not been determined in detail. The purpose of this study was to investigate the effect of silencing of Rac3 expression in human LC cells and the consequences for cell survival. Materials and Methods: Lentivirus small hairpin RNA (shRNA) interference techniques were utilized to knock down the Rac3 gene. Gene and protein expression was quantified by quantitative real-time polymerase chain reaction (qRT-PCR) and Western blotting. LC cell apoptosis was examined by annexin V-APC /propidium iodide staining. Results: Efficient silencing of Rac3 strongly inhibited A549 cell proliferation and colony formation ability, and significantly decreased tumor growth. Moreover, flow cytometry analysis showed that knockdown of Rac3 led to G2/M phase cell cycle arrest as well as an excess accumulation of cells in the $G 1$ and $S$ phase. Conclusions: Thus, functional analysis using shRNAs revealed a critical role for Rac 3 in the tumor growth of $L C$ cells. shRNA silencing of Rac3 could provide an effective strategy to treat LC.
\end{abstract}

Keywords: Lung cancer - Rac3 - proliferation - apoptosis - shRNAs

Asian Pac J Cancer Prev, 16 (7), 3061-3065

\section{Introduction}

Rho-family GTPases are overexpressed in a variety of human cancer and possess a wide range of roles throughout the cell, including the regulation of cell migration and adhesion, mitosis, regulation of kinase activity, and regulation of transcription factors through cell signaling pathways (Baugher et al., 2005; Onesto et al., 2008). Rac proteins are members of the Rho GTPase family and act as molecular switches in regulating a variety of biological response pathways, including cell motility, gene transcription, cell transformation, and cell-cycle progression (Haataja et al., 1997). The Rac family of GTPases consists of three members, Rac1, the splice variant Rac1B, the hematopoietic-cell-specific Rac2 and the most recently described, Rac3, which is particularly strongly expressed in brain (Haataja et al., 1997). These proteins are highly homologous and differ in their transcriptional regulation and tissue distribution. Rac3 differs from other Rac proteins only in the carboxyl terminus, a region essential for subcellular localization and regulatory protein binding (Haataja et al., 1997). As molecular switches, Rac/Rho GTPases cycle between inactive GDP-bound and active GTP-bound states. It has been well studied that activation of RhoGTPases is controlled by guanine nucleotide exchange factor (GEFs) that exchange bound GDP to GTP and by GTPaseactivating proteins (GAPs) that promote GTP hydrolysis.

Rac family members participate in specialized cellular functions. Aberrant activation of Rac3 has been recognized to be crucial in tumor proliferation in both breast cancer and prostate cancer (Engers et al., 2007; Walker et al., 2011). Previous reports implicated that Rac3 may play a paramount role in cell proliferation and cytoskeletal organization (Mira et al., 2000; Chatterjee et al., 2011; Gest et al., 2013). Overexpression of Rac3 may be associated with aggressive tumor behavior. However, studies on Rac3 in LC are scarce.

In the current work, we employed shRNAs silencing of Rac3 in LC cell line. We found that Rac3 knockdown could reduce the LC cells proliferation and induce G0/G1 phase arrest. Our results suggest that Rac3 is essential for the proliferation and tumorigenesis of $\mathrm{LC}$.

\section{Materials and Methods}

\section{Cell lines and cell culture conditions}

Human LC A549 cells were obtained from the Cell Bank of Chinese Academy of Sciences (Shanghai, China). A549 cells were maintained in DMEM medium

${ }^{1}$ Department of Thoracic Surgery, First Affiliated Hospital, China Medical University, , ${ }^{2}$ Department of Thoracic Surgery, Shenyang Chest Hospital, ${ }^{3}$ Department of Thoracic Surgery, General Hospital of Shenyang Military Area Command, Shenyang, Liaoning, China*For correspondence: $237267352 @ q q . c o m$ 
Tieqin Liu et al

(Hyclone) supplemented with $10 \%$ fetal bovine serum (FBS), L-glutamine, penicillin and streptomycin at $37^{\circ} \mathrm{C}$ in a humidified $5 \% \mathrm{CO}_{2}$ atmosphere.

\section{Immunohistochemistry (IHC)}

Primary antibody against Rac3 was blocked and incubated (ProteinTech Group) overnight at $4{ }^{\circ} \mathrm{C}$ and biotinylated secondary antibody for $30 \mathrm{mins}$ at room temperature. After reaction with streptavidin peroxidase conjugate for 10 mins at room temperature, DAB staining was performed.

\section{Lentivirus production}

The complementary DNA sequence of Rac3 was designed from the full-length Rac3 sequence by Shanghai Gene Chem Company (Shanghai, China). After testing knockdown efficiencies, stem-loop oligonucleotides were synthesized and cloned into the lentivirus-based vector PsicoR (Addgene, Boston, MA, USA). A nontargeting scrambled RNA PsicoRvector was generated as a negative control. Lentivirus particles were prepared as described previously (Lois et al., 2002). The lentivirus packaged with GFP was used for the cell proliferation assays, and the lentivirus without GFP was used for the cell apoptosis PI staining to exclude from the interference with GFP signal.

\section{Recombinant LC cells}

LC cells were transfected with the lentivirus-mediated shRNA interference techniques or negative control virus at 7 days and examined at 10 days. Recombinant cells Cells were incubated in serum-free DMEM at $37^{\circ} \mathrm{C}$ for $4 \mathrm{~h}$. Then, 10\% DMEM was added, and the cells were placed in an incubator for additional time (as indicated) for the following experiments.

\section{qRT-PCR analysis}

Total RNA was extracted using Trizol reagent (Invitrogen, Carlsbad, CA, USA) according to the manufacturer's protocol. One microgram of RNA was subjected to reverse transcription. The PCR primers used were as follows: for Rac3, forward 5'- CTCCAAAGTCATCGTCCGGTT and reverse 5'-TGAGTTGCACGTCAAATCTGG, and for GAPDH, forward 5'- TGACTTCAACAGCGACACCCA and reverse 5' - CACCCTGTTGCTGTAGCCAAA. PCR products were separated on a $1 \%$ agarose gel and visualized and photographed under ultra-violet light.

\section{Cell apoptotic assay}

Apoptotic and viable cells fractions were assessed with Annexin V-APC /propidium iodide staining (Invitrogen, USA) on a flow cytometer (Becton, USA) following the manufacturer's instruction. The apoptotic populations were determined by ModFit software. Alternatively, the cells were harvested and washed in PBS, followed by fixation with ice-cold ethanol overnight. The cells were then washed in PBS and incubated in $1 \mathrm{ml}$ staining solution (20 ug/ml propidium iodide and $10 \mathrm{U} / \mathrm{ml}$ RNaseA) for $30 \mathrm{~min}$ at room temperature. The cell cycle distributions were assayed by fluorescence-activated cell sorting using a flow cytometer.

\section{Flow cytometry analysis}

In brief, four days after lentivirus infection, A549 cells were collected, washed by PBS and fixed by $75 \%$ ethanol. Cells were stained with PI and RNase overnight at $4{ }^{\circ} \mathrm{C}$. Samples were then analyzed by Cell Lab Quanta Beckman Coulter. Experiments were repeated at least three times.

\section{MTT assay}

After lentivirus infection, A549 cells were seeded into 96-well plates at a concentration of 2000 cells/ well, respectively. At indicated time points $(1,2,3$, 4, 5 days) at $37^{\circ} \mathrm{C}, 3-(4,5$-dimethylthiazol-2-yl)-2, 5 -diphenyltetrazolium bromide (MTT) solution $(5 \mathrm{mg} / \mathrm{ml}$ ) was added to each well and incubated at $37{ }^{\circ} \mathrm{C}$ for $4 \mathrm{~h}$. Then acidic isopropanol (10\% SDS, $5 \%$ isopropanol and $0.01 \mathrm{M} \mathrm{HCl}$ ) was added to stop the reaction and incubate at $37^{\circ} \mathrm{C}$ for $10 \mathrm{mins}$. The optical density was measured using a microplate reader at the wavelength of $595 \mathrm{~nm}$. Experiments were repeated at least three times.

\section{Western blot analysis}

Four days after lentivirus infection, A549 cells were washed by Phosphate Buffered Saline (PBS) and collected. Whole cell lysis was obtained using $2 \times$ SDS Sample Buffer (100 mM Tris-HCl (pH 6.8), 10 mM EDTA, 4\% SDS, $10 \%$ glycine). Proteins were separated by SDS-PAGE and transferred to PVDF membrane. After the samples were incubated with primary antibodies overnight and secondary antibodies for $2 \mathrm{~h}$ at room temperature, the samples were visualized with enhanced chemiluminescence (Amersham). The following primary antibodies were used in this study: anti-Rac3 (ProteinTech Group); anti-GAPDH (Proteintech Group, Inc., Cat\# 10494-1-AP, dilution 1:100, 000); Experiments were performed in triplicate. The blots were scanned and the band density was measured on the Quantity One imaging software.

\section{Colony formation assay}

To determine the effect of Rac 3 in lung tumorigenesis in vitro, we performed colony formation assay according to the literature (Franken et al., 2006) using a Cellomics Arrayscan (Genechem, Shanghai, China). Briefly, after lentivirus infection, 200 cells of A549 cells were seeded into each well of 6-well plates, respectively. Cells were cultured in the $37^{\circ} \mathrm{C}$ incubator for about 9 days until most single clones have more than 50 cells. Cells were washed by PBS, fixed by $4 \%$ PFA, and then stained with Giemsa. Images were captured by light/florescence microscopy. Experiments were performed at least three times.

\section{Statistical analysis}

Each experiment was repeated at least three times. The results of immunohistochemistry staining were evaluated by $\chi^{2}$ test and the other data were evaluated by Student's $t$ test and expressed as the mean \pm SD. All statistical analyses were performed using SPSS v16.0 software (SPSS, Chicago, IL, USA) and $p<0.05$ was considered statistically significant.

\section{Ethical approval}


All experiments in this manuscript have been performed with the approval of the ethics committee of First Hospital, China Medical University.

\section{Results}

Rac3 is highly expressed in LC cell line

To investigate the function of Rac 3 in $\mathrm{LC}$, we evaluated the expression level of Rac3 in Non small cell lung cancer (NSCLC) cell line A549 by qRT-PCR. We found that Rac3 is highly expressed in A549 cells (Figure 1).

Lentivirus-mediated shRNA efficiently knocks down endogenous expression in A549cells

To investigate the physiological function of Rac 3 in LC cells, we utilized lentivirus-mediated shRNA technology, a powerful method to knock down the endogenous gene expression in vivo. Five days after lentivirus infection, GFP fluorescence demonstrated high percentage of Rac 3 cells being infected by lentivirus. (Figure 2A) Furthermore, using qRT-PCR and western blot analysis, we found that after lentivirus-mediated shRNA, the mRNA level of Rac3 was significantly reduced compared with the control group (Figure 2B). These results suggest that lentivirusmediated shRNA was able to significantly knock down the endogenous Rac3 expression in A549 cells.

Rac3 silencing reduces the proliferation and colony formation ability of A549 cells

Using MTT assay, we examined the impact of Rac3 silencing on the proliferation ability of A549 cells. We found that after lentivirus infection, cell growth was dramatically reduced compared with the control A549 cells (Figure 3A). Since anchorage-independent proliferation is a hallmark of transformed cells, we next examined the ability of Rac 3 to disrupt proliferation in an anchorage-independent environment. The cells were treated with lentivirus-mediated shRNA, re-plated in soft agar, and allowed to form colonies for five weeks. In all cases, silencing of Rac3 reduced the ability of the LC cell line to proliferate in soft agar (Figure 3B). Together, these data indicate that silencing of Rac3 in LC inhibits both anchorage-dependent and -independent proliferation. In summary, Rac3 silencing could inhibit the proliferation and colony formation ability of LC cells in vitro.

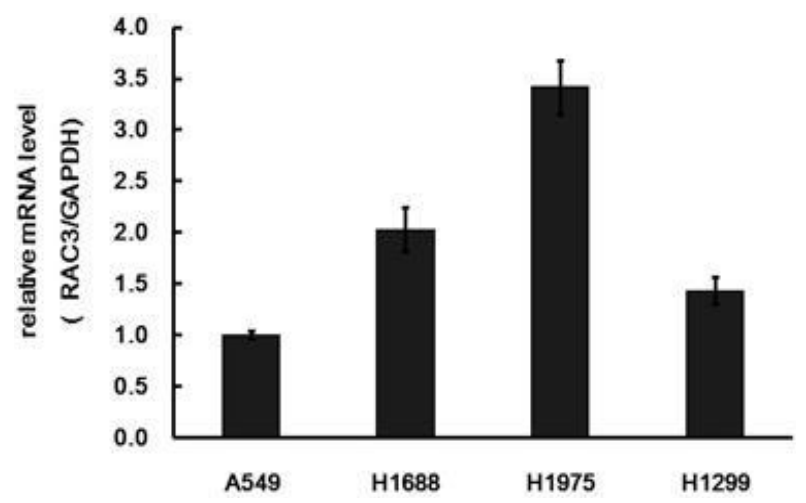

Figure 1. qRT-PCR Analysis of Rac3 Expression in A549 Cells
Rac3 silencing leads to cell cycle arrest

We conducted cell cycle analysis of the A549 cells using flow cytometry. We found that after lentivirusmediated shRNA, $71 \%$ of A549 cells are in the G0/G1 phase and $19 \%$ are in the $\mathrm{S}$ phase of the cell cycle, while $66 \%$ of control infected cells are in the G0/G1 and 19\% are in the S phase of the cell cycle (Figure 4A). The cell cycle analysis revealed that the cell percentage in the G1phase and S phase was increased while the cell numbers in the $\mathrm{G} 2 / \mathrm{M}$ were concomitantly decreased. These results suggested that knockdown of Rac3 in LC cells blocked cell cycle progression.

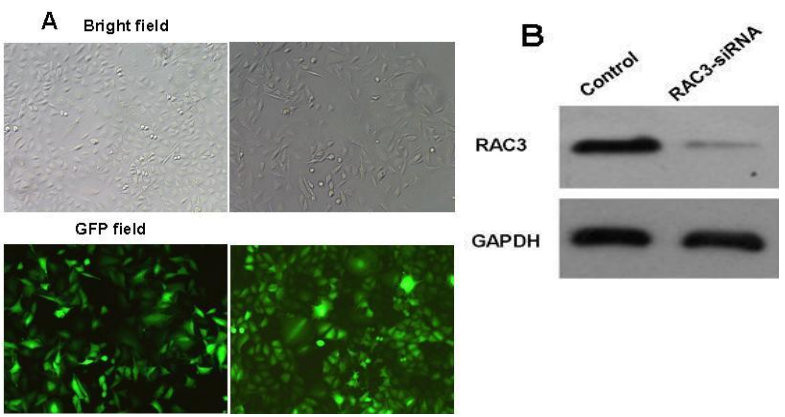

Figure 2. Lentivirus-mediated shRNA decreases Rac3 expression in A549 cells. A: The lentivirus transduction efficiency was estimated 3 days after infection at MOI of 20. GFP expression in transfected cells was observed under light a microscope and a fluorescence microscope. Light micrograph (top panel); fluorescent micrograph (lower panel; x100). B: Western blotting analysis of the knockdown efficiency of Rac3 in A549 cells. Total cellular proteins were extracted 5 days after infection and determined by western blot analysis using antibodies against Rac3 and GAPDH as an internal control. Data represent one out of three separate experiments.

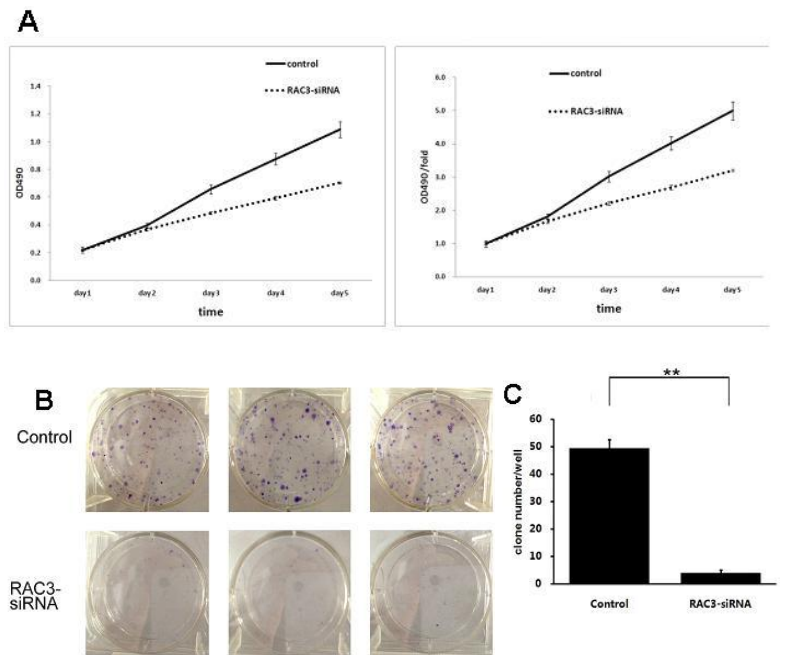

Figure 3. Lentivirus-mediated shRNA Targeting Rac3 Inhibited A549 Cell Proliferation. A: Cell proliferation of untranfected or transfected A549 cells was measured by MTT assay once daily for 5 days. The growth curves showed depletion of Rac3 suppressed LC cell proliferation. B and C: A549 cells were seeded at 200 per well and allowed to form colonies. Colony numbers were counted and recorded. The colonies stained with Giemsa were observed under light microscopy. Data represent the mean $\pm \mathrm{SD}$ of three independent experiments. Depletion of Rac3 disrupted colony formation in LC cells. (**represents $p$ $<0.01$, compared with control.) 


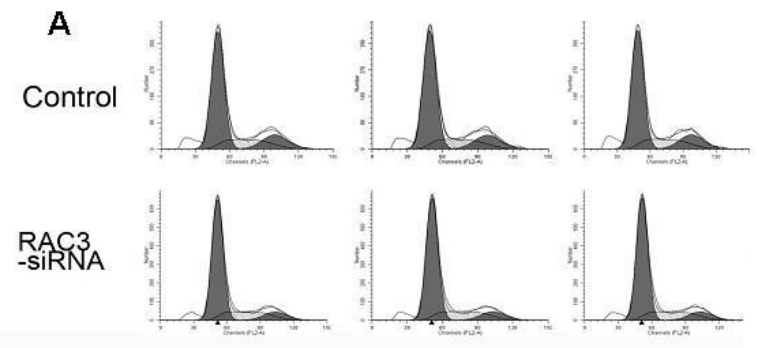

B

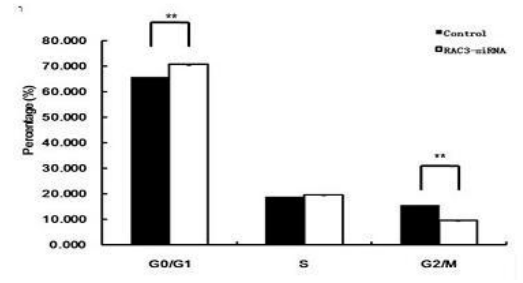

Figure 4. Flow Cytometric Analysis of Cell Cycle Distribution. A and B: Shown are the percentages of cells in the $\mathrm{G} 0 / \mathrm{G} 1, \mathrm{~S}$ and $\mathrm{G} 2 / \mathrm{M}$ phase. Knockdown of Rac3 by shRNA in A549 cells induced cell cycle arrest in G0/G1 phase 5 days after lentivirus transduction. Data represent the mean \pm SD of three independent experiments. (**represents $p<0.01$, compared with control)

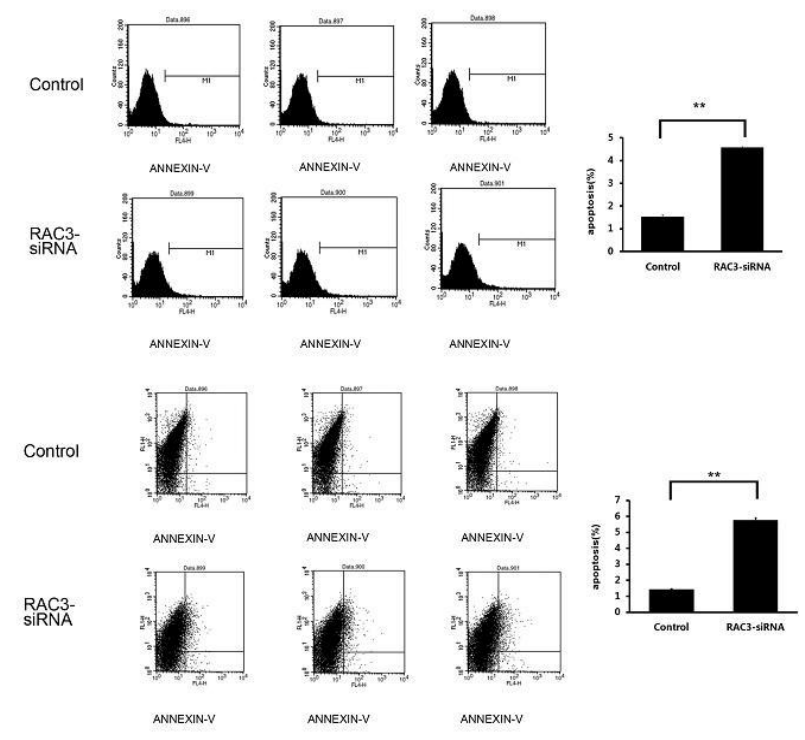

Figure 5. Apoptosis level of A549 Cells was Significantly Increased Compared with the Control Groups after Lentivirus Infection. $(* *$ represents $p<0.01$, compared with control)

Using flow cytometry, we examined the impact of Rac3 silencing on the apoptosis ability of A549 cells. We found that after lentivirus infection, cell apoptosis was dramatically increased compared with the control A549 cells (Figure 5).

\section{Discussion}

Lung cancer is one of the leading causes of cancer death in the world, estimated new LC cases for 2014 total is 224, 210, resulting in 159, 260 deaths (Rebecca et al., 2014). Different therapeutic methods have been used clinically for the treatment of LC, including palliative care, surgery, chemotherapy, radiation therapy and target therapy. However, the 5-year survival is below $20 \%$ worldwide (Allemani et al., 2014). New therapeutic gene targets and methods are needed. Rho GTPases, including Rac1, Rac3, Cdc42, and RhoA, are pivotal regulators of cell morphology, gene expression, cell proliferation, and apoptosis (Manneville et al., 2014). Emerging evidence is beginning to reveal the diverse and profound role of Rac3 in regulating tumor metastasis and the tumorigenic process, raising the possibilities that Rac 3 may be a new therapeutic gene target (Mira et al., 2000; Chatterjee et al., 2011; Gest et al., 2013). RNA interference technique has been widely used to investigate the functional interaction among related genes by silencing selective silence of one gene. The specificity, higher infection rate and stability of this method make it to be a high potential therapeutic method for the treatment of cancer. In the present work, using this method, we specifically suppressed Rac3 expression in LC cell line. Knockdown of Rac3 significantly suppressed the proliferation and colony formation. These observations are well-correlated with the recent studies demonstrating that overexpression of Rac3 plays a crucial role in LC cells progression.

The decrease in proliferation results leads us to question whether the lentivirus-mediated shRNA treated LC cell line was actually accelerating apoptosis. We conducted cell cycle analysis of the A549 cells using flow cytometry. The cell cycle analysis revealed that the cell percentage in the G1phase and S phase was increased while the cell numbers in the G2/M were concomitantly decreased. Apoptosis level of A549 cell was significantly increased compared with the control groups after lentivirus infection. This finding may indicate that Rac3 is both important in LC cell cycle progression and cell survival.

The expression and the role of Rac3 haven't been investigated in LC yet. However, the potential prognostic relevance of Rac expression in human tumors has been discussed. Overexpression of Rac1 protein was considered as an independent predictor of adverse outcome in patients with early operable NSCLC, high Rac1 expression was associated with positive T-stage, and correlated with TNM stage, while no association was observed between Rac1 expression or any other clinicopathologic parameters (Yuan et al., 2009). Silence of Rac 1 expression by shRNA suppressed LC cells migration, invasion and induced rearrangements of the actin cytoskeleton in LC cells (Chen et al., 2011). Also, inhibition of Rac1 expression or disruption of its function significantly reduced cancer cells metastasis in a multitude of tumor models (Chan et al., 2005; Niggli et al., 2009). R Engers et al suggested that increased Rac protein expression in prostate cancer was an independent predictor of decreased disease free survival (DFS) and appeared to result mainly from increased expression of the Rac3 isoform (Engers et al., 2007). However, the mechanism by which Rac3 increases the invasive potential remains to be determined. These findings shed light on the GTPases research in NSCLC.

In conclusion, our results demonstrate that Rac3 silencing by lentivirus- mediated shRNA could inhibit the proliferation of LC cells, which may be due to cell cycle arrest and apoptosis induced. Our studies provide a 
potential therapeutic gene target and a new insight into the development of gene therapy technology to LC treatment.

\section{Acknowledgements}

This reaearch is supported by the National Natural Science Foundation of China (Grant No. 30700821), the Liaoning Bai Qian Wan Talents Program (Grant No. 2011921038), and the Liaoning Province Science and Technology (S and T) Project (Grant No. 2013225585).

\section{References}

Allemani C, Weir HK, Carreira H, et al (2014). Global surveillance of cancer survival 1995-2009: analysis of individual data for 25676887 patients from 279 populationbased registries in 67 countries (CONCORD-2). Lancet. [Epub ahead of print].

Baugher PJ, Krishnamoorthy L, Price JE, et al (2005). Rac1 and Rac3 isoform activation is involved in the invasive and metastatic phenotype of human breast cancer cells. Breast Cancer Res, 7, 965-74.

Chan AY, Coniglio SJ, Chuang YY, et al (2005). Roles of the Rac1 and Rac3 GTPases in human tumor cell invasion. Oncogene, 24, 7821-9.

Chatterjee M, Sequeira L, Jenkins-Kabaila M, et al (2011). Individual rac GTPases mediate aspects of prostate cancer cell and bone marrow endothelial cell interactions. J Signal Transduct, 541851.

Chen QY, Xu LQ, Jiao DM, et al (2011). Silencing of Rac1 modifies LC cell migration, invasion and actin cytoskeleton rearrangements and enhances chemosensitivity to antitumor drugs. Int J Mol Med, 28, 769-76.

Engers R, Ziegler S, Mueller M, et al (2007). Prognostic relevance of increased Rac GTPase expression in prostate carcinomas. Endocr Relat Cancer, 14, 245-56.

Etienne-Manneville S, Hall A (2002). Rho GTPases in cell biology. Nature, 420, 629-35.

Franken NA, Rodermond HM, Stap J, et al (2006). Clonogenic assay of cells in vitro. Nat Protoc, 1, 2315-9.

Gest C, Joimel U, Huang L, et al (2013). Rac3 induces a molecular pathway triggering breast cancer cell aggressiveness: differences in MDA-MB-231 and MCF-7 breast cancer cell lines. BMC Cancer, 13, 63.

Haataja L, Groffen J, Heisterkamp N (1997). Characterization of Rac3, a novel member of the Rho family. J Biol Chem, 272, 20384-88.

Lois C, Hong EJ, Pease S, et al (2002). Germline transmission and tissue-specific expression of transgenes delivered by lentiviral vectors. Science, 295, 868-72.

Mira JP, Benard V, Groffen J, et al (2000). Endogenous, hyperactive RAC3 controls proliferation of breast cancer cells by a p21-activated kinase-dependent pathway. Proc Natl Acad Sci USA, 97, 185-9.

Niggli V, Schlicht D, Affentranger S (2009). Specific roles of Rac1 and Rac2 in motile functions of HT1080 fibrosarcoma cells. Biochem Biophys Res Commun, 386, 688-92.

Onesto C, Shutes A, Picard V, et al (2008). Characterization of EHT 1864, a novel small molecule inhibitor of Rac family small GTPases. Methods Enzymol, 439, 111-29.

Siegel R, Ma J, Zou Z, et al (2014). Cancer statistics, 2014. CA Cancer J Clin, 64, 9-29.

Walker MP, Zhang M, Le TP, et al (2011). RAC3 is a promigratory co-activator of ERalpha. Oncogene, 30, 1984-94.

Yuan K, Qian C, Zheng R (2009). Prognostic significance of immunohistochemical Rac1 expression in survival in early operable non-small cell LC. Med Sci Monit, 15, 313-9. 